\title{
ABRASÃO ULTRASSÔNICA: UMA ALTERNATIVA DE PREPARO CAVITÁRIO PARA O PACIENTE INFANTIL
}

\section{ULTRASONIC ABRASION: AN ALTERNATIVE IN THE PERFORMANCE OF CAVITY PREPARATION IN CHILDHOOD}

\author{
Mariane Hemerly Almeida' \\ Luis Eduardo Paranhos Quintanilha** \\ Fernanda Volpe de Abreu*** \\ Leonardo Santos Antunes"*** \\ Lívia Azeredo Alves Antunes
}

\section{RESUMO}

A técnica da abrasão ultrassônica é realizada com o uso de pontas CVD (Chemical Vapor Deposition), que são formadas através da união química da peça única do diamante à haste metálica, acopladas ao aparelho de ultrassom. Assim, o corte do esmalte e da dentina é feito por oscilação dessas pontas diamantadas e não por ação mecânica, o que promove um desgaste preciso e um preparo conservador da cavidade. Essa técnica proporciona inúmeras vantagens para o profissional e para o paciente, pois diminui a sensação de barulho, pressão, vibração e calor, além de possibilitar um tratamento sem anestesia local e apenas com uso de isolamento relativo, tendo uma boa aceitação pelos pacientes, destacando-se, assim, como aspecto de grande relevância na odontopediatria, para o preparo cavitário. O presente trabalho tem por objetivo descrever um caso clínico utilizando a técnica de abrasão ultrassônica para o preparo cavitário nos dentes 51 e 61 acometidos por lesão cariosa, de um paciente de 04 anos, abordando os aspectos positivos e negativos relacionados a essa técnica.

Descritores: Abrasão ultrassônica, Cárie, Criança, Preparo da cavidade dental.

\section{ABSTRACT}

The technique of ultrasonic abrasion is carried out using tips CVD (Chemical Vapor Deposition), which are formed by the chemical union of one piece of diamond to metal rod coupled to the ultrasonic device. Thus, the cut enamel and dentin is done by oscillation of these diamond burs and not by mechanical action, which promotes an accurate wear and a conservative cavity preparation. This technique provides numerous advantages for the professional and the patient, because it reduces the sensation of noise, pressure, vibration and heat, besides enabling a treatment without local anesthesia and only with the use of relative isolation, with good acceptance by patients, highlighting was thus as an aspect of great relevance in pediatric dentistry for cavity preparation. This paper aims to describe a clinical case using the technique of ultrasonic abrasion for cavity preparation in teeth 51 and 61 affected by carious lesion of a patient of 04 years, addressing the positive and negative aspects to this technique.

Descriptors: Ultrasonic abrasion, Dental carie, Child, Dental cavity preparation

* Graduada em Odontologia pela Faculdade de Odontologia da Universidade Federal Fluminense (FOUFF/NF), Nova Friburgo, Rio de Janeiro, Brasil.

** Professor Associado Universidade Federal Fluminense

*** Doutora em Odontopediatria, Professora Adjunta da Faculdade de Odontologia da Universidade Federal Fluminense (FOUFF/NF), Nova Friburgo, Rio de Janeiro, Brasil - fvolpe07@gmail.com

**** Doutor em Ciências Médicas, Professor Adjunto da Faculdade de Odontologia da Universidade Federal Fluminense (FOUFF/NF), Nova Friburgo, Rio de Janeiro, Brasil - leonardoantunes@id.uff.br

***** Doutora em Odontopediatria, Professora Adjunta da Faculdade de Odontologia da Universidade Federal Fluminense (FOUFF/NF), Nova Friburgo, Rio de Janeiro, Brasil_liviaazeredo@gmail.com 


\section{N T R O D U Ç Ã O}

Pesquisas e novas tecnologias de materiais têm contribuído para a sedimentação de uma Odontologia mais científica, baseada nos conhecimentos biológicos, que traz benefícios aplicáveis à prática clínica, como o desenvolvimento de técnicas menos invasivas para o tratamento de lesões de cárie $^{1-3}$. A abordagem que antes era restauradora e invasiva passou a ser preventiva e de promoção da saúde bucal, preservando a estrutura dental sadia através da mínima intervenção. Portanto, com o melhor entendimento do tratamento da doença cárie, a Odontologia Minimamente Invasiva (OMI) vem ganhando espaço ${ }^{4}$

Dentre as técnicas denominadas minimamente invasivas, a abrasão ultrassônica, realiza o corte da estrutura dentária por vibração, que promove a oscilação de pontas diamantadas em alta frequência, ocasionando um desgaste preciso e um preparo conservador da cavidade ${ }^{5-7}$ diferente da ação mecânica de corte dos sistemas de alta rotação ${ }^{8}$.

No Brasil, as pontas utilizadas nesse sistema são as chamadas pontas CVD (Chemical Vapor Deposition), que são formadas por uma pedra única de diamante através da união química deste a uma haste metálica, proporcionando uma durabilidade até 50 vezes superior às brocas convencionais. Além disso, as pontas podem ser utilizadas em qualquer aparelho de ultrassom, necessitando apenas de um adaptador específico para cada marca ${ }^{6}$.

Alguns estudos vêm demonstrando que esse é um método biologicamente compatível, que não deixa resíduos metálicos, apresentando diversas indicações na dentística, odontopediatria, periodontia e endodontia ${ }^{6,9,10}$. Ademais, essa técnica tem sido relacionada a inúmeras vantagens para o profissional e para o paciente, pois diminui a sensação de barulho, pressão, vibração e calor, além de possibilitar um tratamento sem anestesia local e apenas com uso de isolamento relativo, tendo uma boa aceitação pelos pacientes, destacando-se assim como aspecto de grande relevância na Odontopediatria, para o preparo cavitário ${ }^{10,11}$.

O presente trabalho tem por objetivo descrever um caso clínico utilizando a técnica de abrasão ultrassônica como uma alternativa para o preparo cavitário nos dentes 51 e 61 acometidos por lesão cariosa, de um paciente de 04 anos, abordando os aspectos positivos e negativos relacionados a essa técnica.

\section{RELATO DE CASO}

Um paciente, sexo masculino, saudável, com 4 anos de idade, compareceu à clínica de Odontopediatria da Faculdade de Odontologia da Universidade Federal Fluminense, Nova Friburgo/RJ, acompanhado de seu responsável e com queixa de cáries. Após exame clínico (Figura 1A) e exame radiográfico (Figura $1 \mathrm{~B}$ ) constatou-se a presença de lesão de cárie na mesial dos dentes 51 e 61.

Além disso, ainda no exame radiográfico foi detectada uma reabsorção radicular

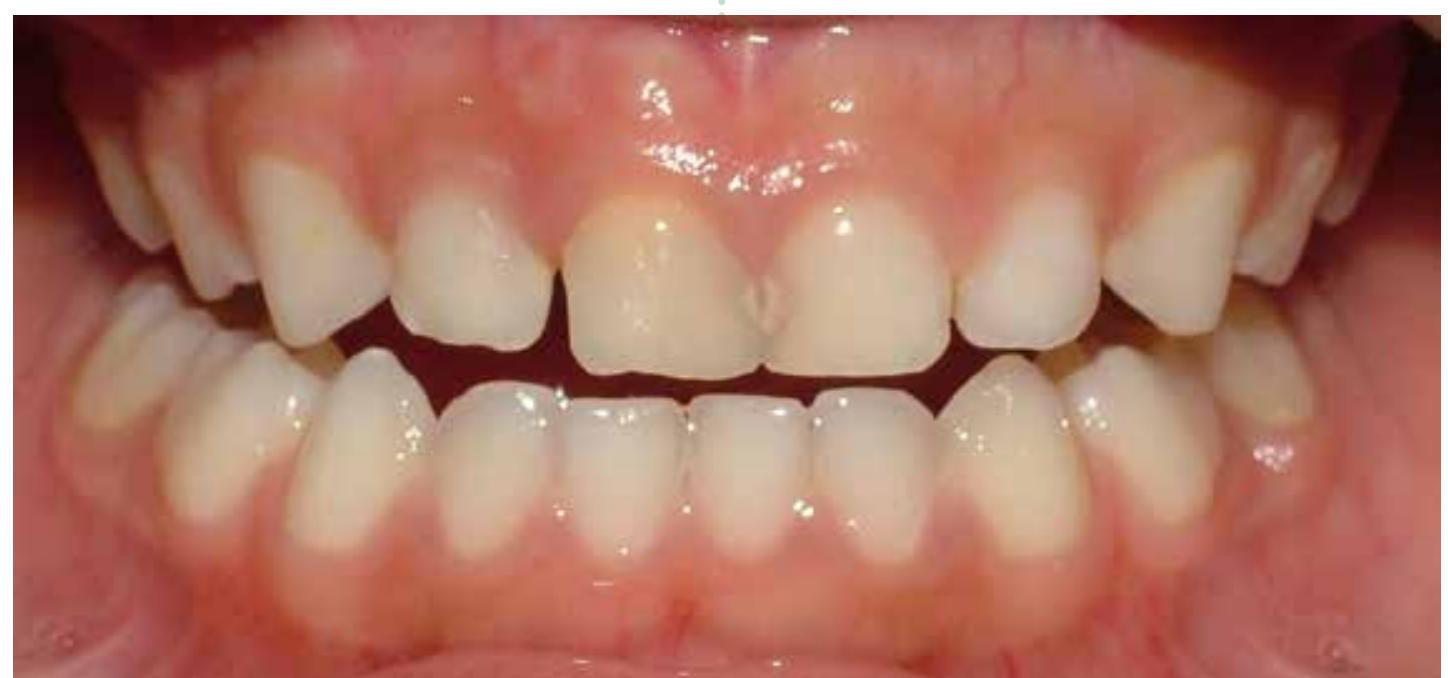

ALMEIDA MH

QUINTANILHA LEP

ABREU FV

ANTUNES LS

ANTUNES LAA

ABRASÃO

ULTRASSÔNICA:

UMA ALTERNATIVA

DE PREPARO

CAVITÁRIO PARA

O PACIENTE

IN FANTIL 
ALMEIDA MH QUINTANILHA LEP

ABREU FV

ANTUNES LS

ANTUNES LAA

ABRASÃO

ULTRASSONNICA:

UMA ALTERNATIVA

DE PREPARO

CAVITÁRIO PARA

O PACIENTE INFANTIL

REV, ODONTOL.

UNIV, CID, SÃO PAULO

$2015 ; 27(1): 56-$

63, JAN-ABR

\section{Figura 1B}

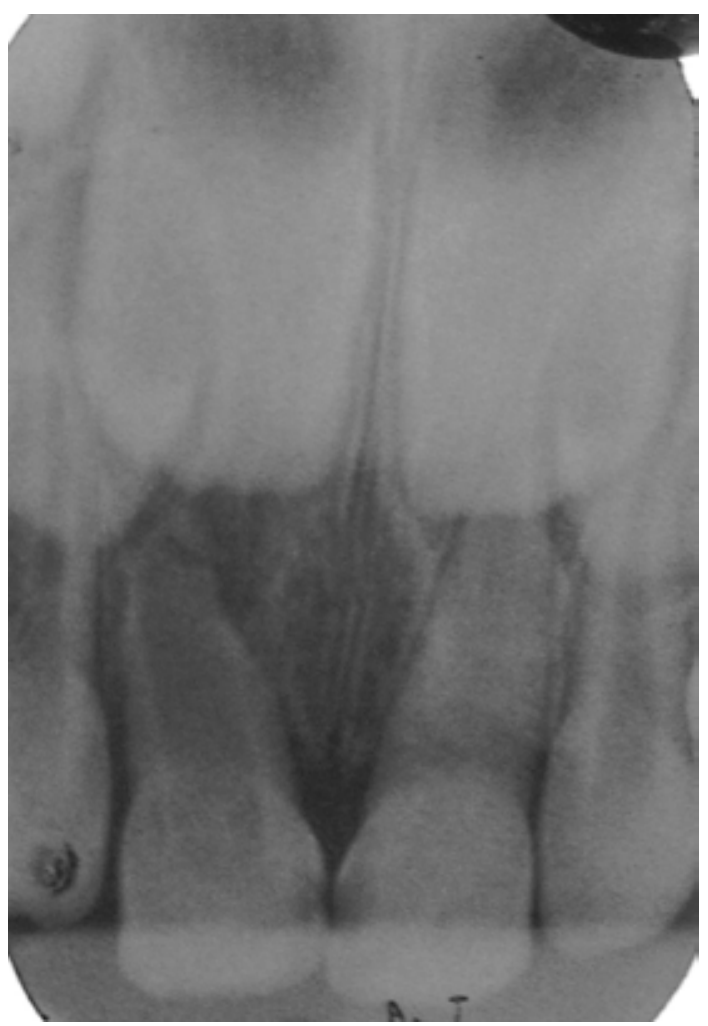

Figura 1: A) Lesão cariosa na mesial dos elementos 51 e 61, observada clinicamente. B) Lesão cariosa avaliada radiograficamente. Observe-se a reabsorção radicular externa no elemento 51.

externa no elemento 61. A lesão cariosa não era tão extensa para ter ocasionado tal sequela radiográfica. Assim, em investigação na anamnese, o responsável relatou que a criança havia sofrido uma queda da própria altura, batendo com a boca numa mesa, há uns 5 meses. Nessa colisão, o responsável relatou que o den- te não havia se movimentado e que havia somente sangrado, sugerindo, segundo informações coletadas, o diagnóstico de uma concussão. Dessa forma, a criança foi encaminhada para o Projeto trauma "UFF Reconstituindo sorrisos" para o tratamento endodôntico do referido elemento dentário (Figura 2).

Em sessão seguinte ao diagnóstico, o tratamento endodôntico foi realizado em sessão única por se tratar de uma biopulpectomia. Como essa criança já havia se submetido a uma sessão cansativa de tratamento endodôntico, optou-se, na $3^{\text {a }}$ sessão, por realizar a restauração das lesões cariosas dos dentes 51 e 61. A técnica escolhida para a remoção do tecido cariado foi a abrasão ultrassônica utilizando pontas diamantadas CVD (CVDentus $®)$, com intuito de realizar um procedimento mais agradável para o jovem paciente.

O procedimento de remoção do tecido cariado e preparo cavitário se iniciou sem a realização de anestesia local. Foi posicionado um afastador spandex infantil ( Prisma ) para manter a criança com a cavidade bucal aberta. A ponta de diamante CVD (CVDentus $\left.{ }^{\circledR}\right)$, esférica de $1,0 \mathrm{x}$ 1,0mm de diâmetro, foi acoplada no aparelho de ultrassom Gnatus ${ }^{\circledR}$ com auxílio de um adaptador.

O aparelho de ultrassom foi regulado com $50 \%$ da potência máxima e não deverá ultrapassar esse valor de acordo com as orientações do fabricante. O fluxo de água foi ajustado de modo que o movimento vibratório da ponta criasse uma

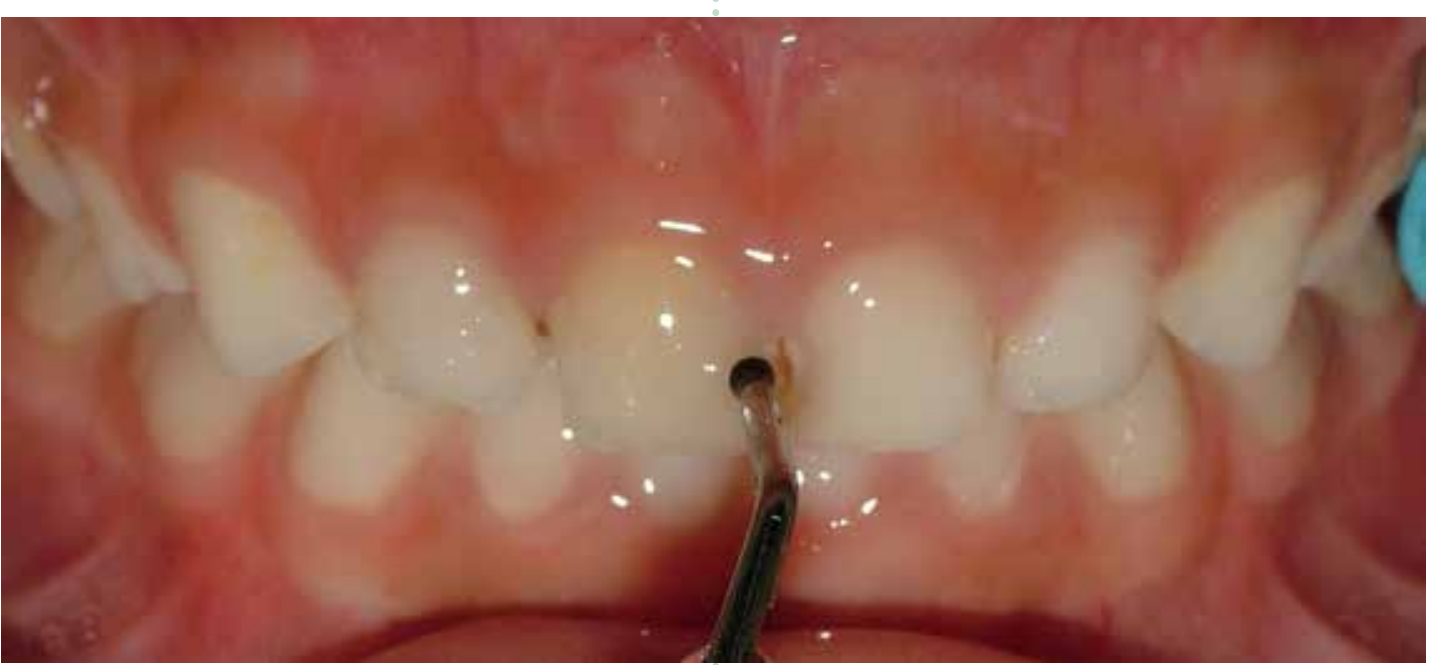

Figura 2: Aspecto radiográfico após tratamento endodôntico. 


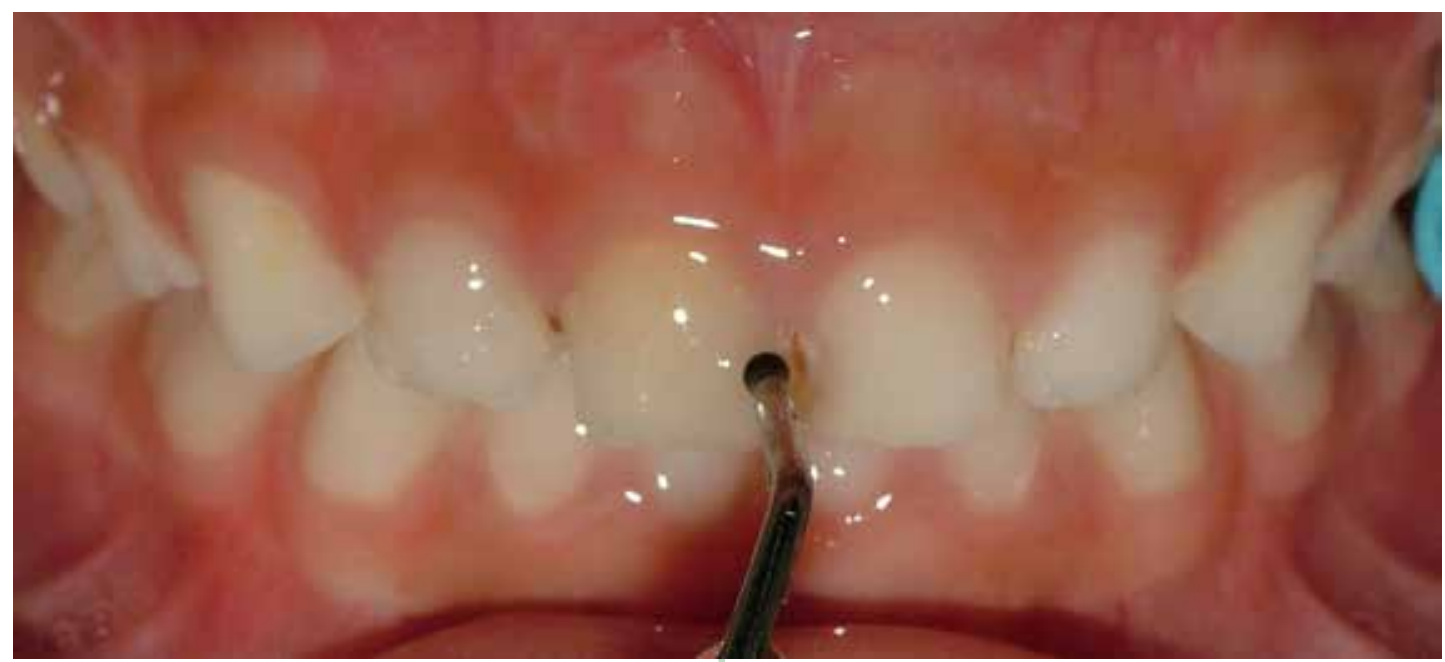

Figura 3: Posicionameto da ponta de diamante CVD (CVDentus®). Observe-se a angulação proporcionada pela ponta, facilitando o acesso ao preparo cavitário
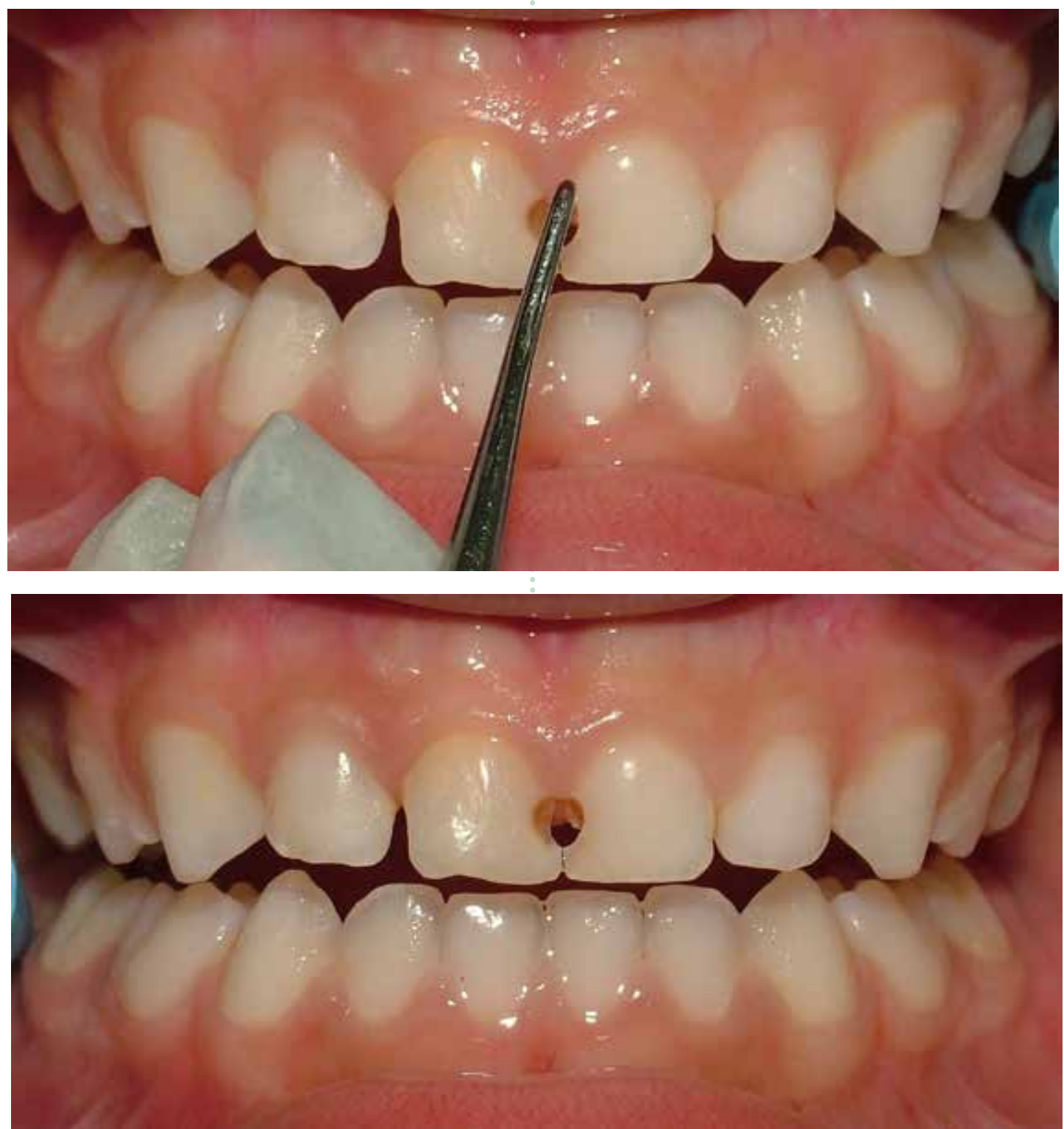

Figura 4: A) Utilização de colher de dentina pàra remoção final do tecido cariado. B) Preparo cavitário finalizado. Observe-se a preservação de estrutura dental.

ALMEIDA MH

QUINTANILHA LEP

ABREU FV

ANTUNES LS

ANTUNES LAA

ABRASÃO

ULTRASSONNICA:

UMA ALTERNATIVA

DE PREPARO

CAVITÁRIO PARA

o PACIENTE

INFANTIL

$\therefore 59 \ldots$

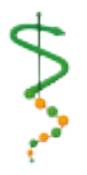

REV. OdONTOL.

Univ. Cid. São

PAULO

2015; 27(1): 56 -

63, JAN-ABR 
ALMEIDA MH QUINTANILHA LEP

ABREU FV

ANTUNES LS

ANTUNES LAA

$A B R A S \tilde{A} O$

ULTRASSONNICA:

UMA ALTERNATIVA

DE PREPARO

CAVITÁRIO PARA

O PACIENTE

INFANTIL
REV, ODONTOL,

UNIV, CID, SÃO

PAULO

$2015 ; 27(1): 56-$

63, JAN-ABR

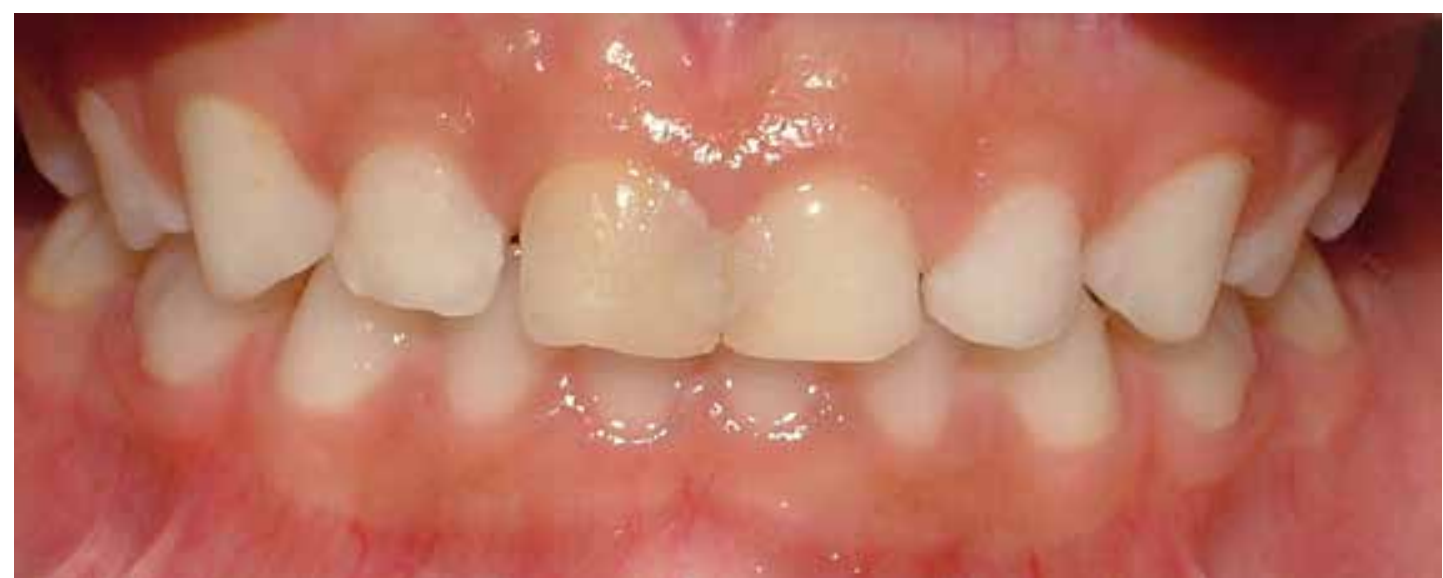

Figura 5: Aspecto final, após restauração com resina composta.

névoa de partículas de água ao redor da mesma $^{12}$. A ponta de sucção foi utilizada durante todo o processo. A angulação proporcionada pela ponta facilitou o acesso ao preparo cavitário. Os preparos foram realizados com movimentos firmes e suaves, proporcionando corte por "oscilação" (Figura 3).

De acordo com o preconizado por Vieira e Viera (2002), utilizou-se escavador manual como a colher de dentina ( $\mathrm{Fi}$ gura 4A) para a conclusão da remoção do tecido cariado, preservando-se estrutura dental sadia (Figura 4B).

Para o procedimento restaurador, $\mathrm{O}$ paciente foi mantido com o afastador Spandex (Prisma), sucção contínua e isolamento relativo. Os dentes foram lavados

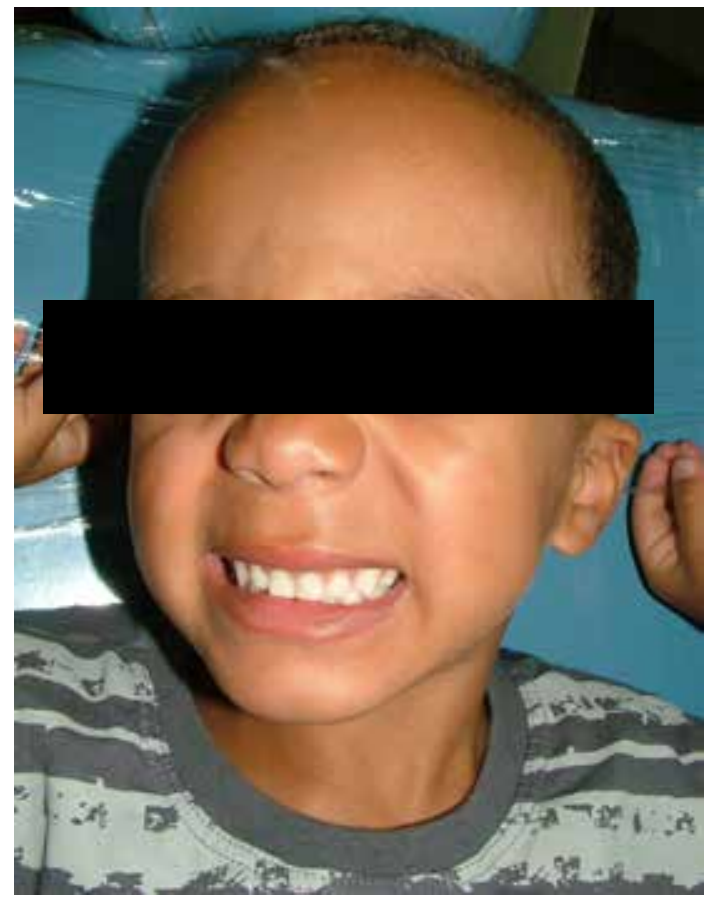

Figura 6: Satisfação do paciente imediatamente após a restauração. com jatos de ar/água. Após a remoção do excesso de umidade, o esmalte foi condicionado com ácido fosfórico 37\% (Condac $37^{\circledR}$, FGM) por 15 segundos e a dentina por 07 segundos. O ácido foi removido em seguida por lavagem por 10 segundos. O excesso de umidade da dentina foi removido com pequenos discos de papel filtro, enquanto o esmalte foi seco com jatos de ar até que apresentasse superfície opaca. A primeira camada do adesivo Adper ${ }^{\mathrm{TM}}$ Single Bond 2 foi aplicada e esfregada vigorosamente com microbrush (DFL) ao longo de todas as superfícies cavitárias por 20 segundos. Após a aplicação, um leve jato de ar foi aplicado à distância por 05 segundos para aumentar ainda mais a evaporação do solvente. Outra camada foi aplicada da mesma forma que a primeira e as duas fotoativadas por 10 segundos com unidade fotoativadora baseada em LED. A resina composta Filtek $^{\mathrm{TM}}$ Z250 (3M ESPE) foi inserida em incrementos de 2.0 milímetros de modo a recuperar o contorno anatômico original, perdido em função do avanço da lesão cariosa (Figura 5). Concluída a restauração (Figura 5), o acabamento foi realizado com pontas diamantadas tronco cônicas de $0,4 \times 0,3 \mathrm{~mm}$ de diâmetro, da série de acabamento CVD (CVDentus $\left.{ }^{\circledR}\right)$, acopladas no mesmo aparelho de ultrassom regulado com $30 \%$ da potência máxima. E o polimento da restauração foi realizado com pontas de silicone (Enhance ${ }^{\circledR}$, Dentsply) e pasta diamantada para polimento (Diamond Excel ${ }^{\mathrm{TM}}, \mathrm{FGM}$ ).

Não foram relatados pelo paciente nenhuma queixa, dor ou desconforto durante a preparação da cavidade. Ao final do 
procedimento (Figura 6), pôde-se observar a satisfação do paciente pela consulta rápida e agradável, haja vista que, em consulta prévia, o paciente havia passado por uma consulta de extrema tensão: o tratamento endodôntico. Todo o tratamento teve o consentimento dos responsáveis e foi aprovado pelo Comitê de Ética em Pesquisa da Faculdade de Medicina do Hospital Universitário Antônio Pedro, CAAE 0016.0.258.000-10

\section{I SCUSSÃO}

Na Odontopediatria, as crianças ficam ansiosas, desconfortáveis e nervosas durante o atendimento odontológico, sendo os fatores causadores a anestesia, o baruIho do motor da alta rotação e o medo de ser submetido a um procedimento doloroso. Portanto, se a reação da criança for desproporcional à situação vivida no consultório, pode ter um efeito negativo no tratamento odontológico, uma vez que o grau de cooperação e aceitação das crianças, durante o procedimento de remoção de tecido cariado, tem influência direta no desempenho clínico e sucesso do tratamento odontopediátrico ${ }^{13}$.

Por isso, o profissional que trabalha com crianças deve recorrer a técnicas de manejo de comportamento e psicologia infantil para que o tratamento seja realizado corretamente e de maneira segura ${ }^{14}$. Aliado a essas técnicas, o conhecimento de métodos alternativos que possam ajudar nesse controle do comportamento do paciente é de extrema importância para aqueles que lidam com crianças.

O sistema ultrassônico é bem aceito pelos pacientes. De acordo $\operatorname{com}^{13}$, a metade das crianças tinha medo ou ficava ansiosa e nervosa durante o atendimento odontológico. Os fatores que mais geravam medo eram a anestesia e a visão do motor $^{13}$, e o autor ainda relatou que, embora os responsáveis tivessem pouco conhecimento sobre os métodos alternativos de remoção de cárie, como a abrasão ultrassônica, eles demonstraram receptividade à utilização dos mesmos em seus filhos.

Outras características que foram observadas como pontos positivos durante a execução do caso com a técnica proposta são relatadas por diversos autores como: permitir menor desconforto ${ }^{6,12}$, possibilidade da sua utilização sem anestesia local 6,15, diminuição da sensação de barulho, pressão, vibração e calor bem como sua execução apenas com uso de isolamento relativo. Com essas vantagens, as crianças se sentem mais à vontade e menos ansiosas no ambiente odontológico ${ }^{16}$.

No presente caso, a criança passou por um procedimento tenso previamente à consulta de remoção do tecido cariado. Dessa forma, o paciente estava receoso para a execução desta consulta subsequente. De acordo com $^{17}$, as crianças que necessitam de retreinamento como abordagem apresentam considerável apreensão ou comportamento negativo. Assim, com base no proposto por esses autores, iniciar um programa de retreinamento que conduz à modelagem do comportamento foi a opção neste caso. A utilização da técnica de abrasão ultrassônica foi de grande importância para a readaptação do comportamento da criança perante os procedimentos odontológicos devido às suas características altamente positivas.

A técnica de abrasão ultrassônica é bem simples, entretanto, a manipulação dos instrumentos em preparos cavitários difere da utilizada com padrão rotatório ${ }^{6,12}$, exigindo, portanto, prévio treinamento do profissional. Segundo os trabalhos ${ }^{5,15}$, os instrumentos ultrassônicos promovem preparos conservadores, devido aos cortes de alta precisão. Em algumas circunstâncias, porém, escavadores manuais em forma de colheres de dentina devem ser utilizados, conforme executados no presente caso, para remoção de tecidos cariados amolecidos remanescentes ${ }^{5,12}$, já que nessas estruturas ${ }^{6}$ e em cavidades extensas a técnica está contraindicada.

De acordo $\mathrm{Com}^{18}$ as pontas CVD apresentaram maior durabilidade e qualidade de acabamento, facilidade de limpeza e garantia do contato do dente apenas com o diamante, quando comparadas às pontas convencionais. Essas qualidades oferecem novas perspectivas na operacionalidade e qualidade do trabalho. Os resultados evidenciaram que os preparos cavitários em esmalte e dentina confeccionados com
ALMEIDA MH

QUINTANILHA LEP

ABREU FV

ANTUNES LS

ANTUNES LAA

ABRASÃO

ULTRASSONNICA:

UMA ALTERNATIVA

DE PREPARO

CAVITÁRIO PARA

O PACIENTE

INFANTIL

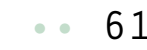

REV, ODONTOL.

UNIV, CID, SÃo

PAULO

2015; 27(1): 56 -

63, JAN-ABR 
ALMEIDA MH QUINTANILHA LEP ABREU FV ANTUNES LS ANTUNES LAA

ABRASÃO

ULTRASSONNICA: UMA ALTERNATIVA DE PREPARO CAVITÁRIO PARA O PACIENTE INFANTIL

REV, ODONTOL.

UNIV. CID, SÃO PAULO

$2015 ; 27(1): 56-$ 63, JAN-ABR ponta de diamante CVD em ultrassom foram mais conservadores do que os preparos com a ponta diamantada em alta rotação ${ }^{18}$. Esse fato pode ser observado no caso apresentado com remoção conservadora de tecido cariado.

A implementação da técnica de abrasão ultrassônica pode também estar relacionada a uma melhor aderência dos materiais restauradores. É sabido que as superfícies criadas por preparos cavitários podem ser significantes para adaptação material restaurador utilizado nas restaurações adesivas, uma vez que podem prevenir a microinfiltração ${ }^{19}$. De acordo com ${ }^{6}$, preparos em dentes decíduos, confeccionados a partir das pontas CVD, apresentam menor quantidade de smear layer, facilitando a limpeza e o condicionamento ácido. Todavia, esse achado foi contestado por ${ }^{20}$, ao afirmarem que, embora os preparos com as pontas CVD tenham propiciado menor formação de estrias, observou-se presença marcante de smear layer com túbulos dentinários obstruídos em ambos os grupos avaliados: alta rotação e ultrassom. Esse fato sugere a importância de mais estudos nessa área e de enfatizar ou refutar esses resultados como, por exemplo, estudos clínicos como sugeridos por ${ }^{21}$

Ademais, a abrasão ultrassônica ainda apresenta algumas limitações como: pouca disponibilidade das pontas CVD no mercado, a lenta ação de corte. A primeira limitação torna esse sistema ainda pouco acessível e desconhecido. No entanto, é aí que se nota a importância da divulgação de relatos de sucesso e a praticidade como a do presente caso, para que estimulem sua utilização pelos clínicos em geral. O segundo ponto abordado em relação à ação lenta de corte é uma limitação que preocupa na execução e casos em pacientes infantis, uma vez que, na odontopediatria, são preconizadas consultas curtas para não deteriorar o comportamento dos pacientes infantis. Vieira et al. ${ }^{11}$ (2007) realmente comprovaram que o tempo de preparo cavitário realizado pela alta rotação, em estudo in vitro, foi mais rápido do que nos preparos com abrasão ultrassônica. No entanto, essa limitação pode ser administrada e superada pelo fato de que, nessa técnica, ao ser executada clinicamente, não é necessário anestesiar nem isolar e compensa o tempo clínico dispendido no preparo cavitário.

Assim, com base na discussão proposta, observa-se que o sistema de abrasão ultrassônica utilizando pontas diamantadas CVD parece ser uma alternativa promissora de preparo cavitário para a clínica em geral e, principalmente, para pacientes infantis.

No presente caso, pode-se constatar total sucesso com finalização do procedimento com a completa satisfação da criança e dos responsáveis, uma vez que não houve queixa de dor ou desconforto durante o procedimento pelo paciente. Porém, estudos clínicos longitudinais devem ser realizados, para confirmar as características satisfatórias do sistema, assim como sugerir aos fabricantes o aperfeiçoamento das deficiências.

\section{CONCLUSÃO}

A técnica de abrasão ultrassônica com pontas diamantadas CVD foi adequado para a remoção de tecido cariado no caso proposto, demonstrando-se bastante útil na Odontopediatria, uma vez que é uma técnica mais agradável, podendo ser utilizada para remodelação do comportamento do paciente. 


\section{REFERÊNCIAS}

1. Tyas MJ, Anusavice KJ, Frencken JE, Mount GJ. Minimal intervention dentistry--a review. FDI Commission Project 1-97. Int Dent J 2000 Feb;50(1):1-12.

2. Yazici AR, Ozgunaltay G, Dayangac B. A scanning electron microscopic study of different caries removal techniques on human dentin. Oper Dent 2002 Jul-Aug;27(4):360-6.

3. Cehreli ZC, Yazici AR, Akca T, Ozgunaltay G. A morphological and microtensile bond strength evaluation of a single-bottle adhesive to caries-affected human dentine after four different caries removal techniques. J Dent 2003 Aug;31(6):429-35.

4. Maia LC, Buczynski AK. Odontologia minimamente invasiva. In: Maia LC, Primo LG, editors. Odontologia integrada na infância. São Paulo: Santos; 2012 p. 17-193.

5. Banerjee A, Watson TF, Kidd EA. Dentine caries excavation: a review of current clinical techniques. Br Dent J 2000 May $13 ; 188(9)$ :476-82.

6. Vieira D. Pontas de diamante CVD: início do fim da alta rotação? . J Am Dent Assoc Brasil 2002 5(10):307-13.

7. Wicht MJ, Haak R, Fritz UB, Noack MJ. Primary preparation of class II cavities with oscillating systems. Am J Dent 2002 Feb;15(1):21-5.

8. Crawford PR. The birth of the bur (and how a Canadian changed it all!). J Can Dent Assoc 1990 Feb;56(2):123-6.

9. Carvalho CA, Fagundes TC, Barata TJ, Trava-Airoldi VJ, Navarro MF. The use of CVD diamond burs for ultraconservative cavity preparations: a report of two cases. J Esthet Restor Dent 2007 19(1):19-28;

10. Antunes LAA, Antunes L, Mai L. Diamond used in dentistry. Industrial Diamond Review 2007 4(8)

11. Vieira ASB, Antunes LAA, Maia LC, Primo L. Abrasão ultra-sônica: uma alternativa para a confecção de preparos cavitários. Pesq Bras Odontoped Clin Integr 2007 maio/ago;7(2):181-86.
12. Postle H. Ultrasonic cavity preparation. Journal of Prosthetic Dentistry 1958 Jan;8(1):153-60.

13. Lopes F, Pedro RL, Antunes LA, Pomarico L, Maia LC. Percepção de responsáveis sobre medo odontológico e técnica alternativa para remoção de cárie. Rev Bras Odontol 2011 jul/dez;68(2):16670.

14. Ramos-Jorge ML. Comportamento infantil no ambiente odontológico: aspectos psicológicos e sociais. J Bras Odontopediatr Odontol Bebe 2003 jan./fev.;6(9):70-4.

15. Laird WR, Walmsley AD. Ultrasound in dentistry. Part 1--Biophysical interactions. J Dent 1991 Feb;19(1):14-7.

16. Mastrantonio SDS, Gondim JO, Josgrilberg ÉB, Cordeiro RCL. Redução do medo durante o tratamento odontológico utilizando pontas ultrassônicas. RGO - Revista Gaúcha de Odontologia 2010 58(1):119-22.

17. Dean JÁ, Avery DR, Mcdonald RE. Odontopediatria para crianças e adolescentes. 9 ed. Rio de Janeiro: Guanabara; 2011.

18. Lima LM, Motisuki C, Corat EJ, Santos-Pinto L. Comparative cutting effectiveness of an ultrasonic diamond tip and a high-speed diamond bur. Minerva Stomatol 2009 Mar;58(3):93-8.

19. Nozaka K, Suruga Y, Amari E. Microleakage of composite resins in cavities of upper primary molars. Int J Paediatr Dent 1999 Sep;9(3):185-94.

20. Vieira AS, dos Santos MP, Antunes LA, Primo LG, Maia LC. Preparation time and sealing effect of cavities prepared by an ultrasonic device and a highspeed diamond rotary cutting system. J Oral Sci 2007 Sep;49(3):207-11.

21. Carvalho FP, Kobayashi TY, Rios D, Oliveira TM, Machado MAAMM, Silva SMB. Uso de ponta diamantada Cvdentus ${ }^{\circledR}$ para preparo cavitário em dentes decíduos: estudo In Vivo. Pesqui Bras Odontopediatria Clín Integr 2012 mar;11(3):459-63.

Recebido em 01/02/2015

Aceito em 11/03/2015
ALMEIDA MH

QUINTANILHA LEP

ABREU FV

ANTUNES LS

ANTUNES LAA

ABRASÃO

ULTRASSONNICA:

UMA ALTERNATIVA

DE PREPARO

cavitário PARA

o PACIENTE

IN FANTIL

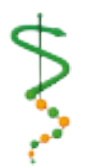

REV, ODONTOL.

UNIV. CID. SÃO

PAULO

2015; $27(1): 56$ -

63, JAN-ABR 